TRANSACTIONS OF THE

AMERICAN MATHEMATICAL SOCIETY

Volume 357, Number 12, Pages 5051-5066

S 0002-9947(05)03686-X

Article electronically published on March 31, 2005

\title{
FILTRATIONS IN SEMISIMPLE RINGS
}

\author{
D. S. PASSMAN
}

\begin{abstract}
In this paper, we describe the maximal bounded $\mathbb{Z}$-filtrations of Artinian semisimple rings. These turn out to be the filtrations associated to finite $\mathbb{Z}$-gradings. We also consider simple Artinian rings with involution, in characteristic $\neq 2$, and we determine those bounded $\mathbb{Z}$-filtrations that are maximal subject to being stable under the action of the involution. Finally, we briefly discuss the analogous questions for filtrations with respect to other Archimedean ordered groups.
\end{abstract}

\section{INTRODUCTION}

Let $R$ be a ring with 1 . A $\mathbb{Z}$-filtration $\mathcal{F}=\left\{F_{i} \mid i \in \mathbb{Z}\right\}$ of $R$ is a collection of additive subgroups

$$
\cdots \subseteq F_{-2} \subseteq F_{-1} \subseteq F_{0} \subseteq F_{1} \subseteq F_{2} \subseteq \cdots
$$

indexed by the integers $\mathbb{Z}$ such that $F_{i} F_{j} \subseteq F_{i+j}$ for all $i, j \in \mathbb{Z}$. One usually also assumes that $\bigcup_{i} F_{i}=R$ and $\bigcap_{i} F_{i}=0$. We say that the filtration is bounded if there exist integers $\ell$ and $\ell^{\prime}$ with $F_{\ell}=0$ and $F_{\ell^{\prime}}=R$. If $K$ is a field and $R=\mathrm{M}_{n}(K)$ is the ring of $n \times n$ matrices over $K$, then every $n$-dimensional $K$ algebra $A$ embeds in $R$ and gives rise to a filtration with $F_{-1}=0, F_{0}=A$ and $F_{1}=R$. Thus, it is hopeless to attempt to classify all the bounded filtrations of such a matrix ring, even under the additional assumption that the $F_{i}$ are $K$-vector spaces. Nevertheless, there is at least something that can be done.

Again, let $\mathcal{F}$ be a filtration of an arbitrary $\operatorname{ring} R$. If $\mathcal{G}=\left\{G_{i} \mid i \in \mathbb{Z}\right\}$ is a second such filtration, we say that $\mathcal{G}$ contains $\mathcal{F}$, or $\mathcal{G}$ is larger than $\mathcal{F}$, if $G_{i} \supseteq F_{i}$ for all $i$. In particular, it makes sense to speak about maximal bounded filtrations, and it turns out that these filtrations can be classified when $R$ is a semisimple Artinian ring. Indeed, this is the content of Theorem 3.6 and it follows that these are precisely the filtrations associated to finite $\mathbb{Z}$-gradings of $R$. We note that maximal bounded filtrations appear, possibly for the first time, in $[\mathrm{B}]$.

In this paper, we also study simple Artinian rings $R$, in characteristic $\neq 2$, admitting an involution $*$. Here we consider $*$-filtrations, namely ordinary filtrations $\mathcal{F}=\left\{F_{i} \mid i \in \mathbb{Z}\right\}$ with the additional property that each $F_{i}$ is $*$-stable. Again, we can speak of bounded $*$-filtrations and then of $*$-filtrations maximal in the collection of bounded $*$-filtrations. These $*$-maximal filtrations are classified in Theorem 5.5. In both cases, the proof is a two-step process. The first step requires that we find a

Received by the editors October 29, 2003 and, in revised form, March 16, 2004.

2000 Mathematics Subject Classification. Primary 16W70, 16P20, 16W10.

The author's research was supported in part by NSA grant 144-LQ65. He would also like to thank Yiftach Barnea for interesting conversations on this problem.

(C)2005 American Mathematical Society 
full diagonal in $F_{0}$, with the diagonal stable under the action of $*$ when we consider rings with involution. In the second step, we classify those maximal filtrations with $F_{0}$ containing a fixed diagonal.

In the last section of this paper, we briefly consider filtrations with respect to other Archimedean ordered groups. These groups are, of course, all additive subgroups of the real numbers $\mathbb{R}$ and hence are either dense or isomorphic to $\mathbb{Z}$. The following lemma applies equally well to filtrations over dense subgroups, and with the same proof, but we state it only for filtrations with group $\mathbb{Z}$.

Lemma 1.1. Let $R$ be a semiprime ring and let $\mathcal{F}=\left\{F_{i} \mid i \in \mathbb{Z}\right\}$ be a bounded filtration of $R$. Then $\mathcal{F}$ is contained in a maximal bounded filtration. If, in addition, $R$ admits an involution $*$ and $\mathcal{F}$ is a $*$-filtration, then $\mathcal{F}$ is contained in a maximal bounded $*$-filtration.

Proof. Let $\mathcal{F}=\left\{F_{i} \mid i \in \mathbb{Z}\right\}$ be given with $F_{a-1}=R$ and suppose $\mathcal{G}=\left\{G_{i} \mid i \in \mathbb{Z}\right\}$ is any bounded filtration containing $\mathcal{F}$. Then $G_{a-1}=R$ and $G_{-a} R=G_{-a} G_{a-1} \subseteq$ $G_{-1}$. But $\mathcal{G}$ is bounded, so $G_{-1}$ is nilpotent and hence $G_{-a} R$ is a nilpotent right ideal in the semiprime ring $R$. We conclude that $G_{-a}=0$ and, with this, Zorn's Lemma yields the result.

Of course, filtrations often arise from gradings and bounded filtrations arise from finite gradings. Recall that $R=\bigoplus \sum_{i \in \mathbb{Z}} R_{i}$ is a $\mathbb{Z}$-grading of the ring if each $R_{i}$ is an additive subgroup with $R_{i} R_{j} \subseteq R_{i+j}$ for all $i, j$. Furthermore, this grading is said to be finite if only finitely many of the components $R_{i}$ are nonzero. In this case, it is clear that each $R_{i}$ with $i \neq 0$ is nilpotent. Now any such grading determines an associated $\mathbb{Z}$-filtration $\mathcal{F}=\left\{F_{i} \mid i \in \mathbb{Z}\right\}$ by defining $F_{i}=\sum_{j \leq i} R_{j}$, and we have

Lemma 1.2. Let $R$ be a semiprime ring with a finite $\mathbb{Z}$-grading given by $R=$ $\bigoplus \sum_{i} R_{i}$. If $\mathcal{F}=\left\{F_{i} \mid i \in \mathbb{Z}\right\}$ is the filtration associated to this grading, then $\mathcal{F}$ is a maximal bounded filtration.

Proof. By assumption, only finitely many of the $R_{i}$ are nonzero. Hence, since $F_{i}=\sum_{j \leq i} R_{j}$, it is clear that $\mathcal{F}$ is at least a bounded filtration. Suppose, by way of contradiction, that $\mathcal{G}=\left\{G_{i} \mid i \in \mathbb{Z}\right\}$ is a bounded filtration properly larger than $\mathcal{F}$, and define $X_{i}=G_{i-1} \cap R_{i}$. Of course, only finitely many of these can be nonzero. Furthermore, since $R_{k} \subseteq F_{k} \subseteq G_{k}$, we see that $R_{k} X_{i} \subseteq X_{i+k}$ and $X_{i} R_{k} \subseteq X_{i+k}$. Note also that each $X_{i}$ is nilpotent. Indeed, this follows if $i>0$ since $X_{i} \subseteq R_{i}$, and it follows if $i \leq 0$ since $X_{i} \subseteq G_{i-1} \subseteq G_{-1}$.

We claim that some $X_{i}$ is not zero. Indeed, since $\mathcal{G}$ is properly larger than $\mathcal{F}$, we have $G_{t}$ properly larger than $F_{t}$ for some $t$, and hence we can choose $r \in R$ with $r \in G_{t} \backslash F_{t}$. Write $r=\sum_{i} r_{i} \in \sum_{i} R_{i}$ as the sum of its homogeneous components, and let $n$ be the maximal subscript with $r_{n} \neq 0$. Since $r \notin F_{t}$, we must have $n>t$. Then $r \in G_{n-1}$ and $r-r_{n} \in \sum_{i \leq n-1} R_{i}=F_{n-1} \subseteq G_{n-1}$, so $0 \neq r_{n} \in G_{n-1} \cap R_{n}=X_{n}$. We can now choose subscript $a$ minimal with $X_{a} \neq 0$.

Since $R$ is semiprime, we have $X_{a} R X_{a} \neq 0$ and hence $X_{a} R_{k} X_{a} \neq 0$ for some $k$. Since $X_{a} R_{k} \subseteq X_{a+k}$ and $R_{k} X_{a} \subseteq X_{a+k}$, it follows that $X_{a+k} X_{a} \neq 0$ and $X_{a} X_{a+k} \neq 0$. We can now choose $b$ maximal among all subscripts $\ell$ with either $X_{\ell} X_{a} \neq 0$ or $X_{a} X_{\ell} \neq 0$ or both. Say $X_{a} X_{b} \neq 0$.

If $j<0$, then $R_{j} X_{a} \subseteq X_{j+a}=0$ and $X_{a} R_{j} \subseteq X_{j+a}=0$ by the minimality of $a$. Furthermore, if $j>0$, then $X_{b} R_{j} X_{a} \subseteq X_{b+j} X_{a}=0$ and $X_{a} R_{j} X_{b} \subseteq X_{a} X_{b+j}=0$ by the maximality of $b$. Since $R=\sum_{j} R_{j}$ and $1 \in R_{0}$, it now follows that $X_{a} R X_{b}=$ 
$X_{a} R_{0} X_{b}=X_{a} X_{b} \neq 0$ and $X_{b} R X_{a}=X_{b} X_{a}$. In particular, $I=R X_{a} R X_{b}$ is a nonzero left ideal of $R$, and the previous formulas show that all internal $R$-factors in the power $I^{m}$ can be deleted. In other words, $I^{m}=\left(R X_{a} R X_{b}\right)^{m}=R\left(X_{a} X_{b}\right)^{m}$. But $X_{a} X_{b} \subseteq G_{a+b-2} \cap R_{a+b} \subseteq G_{a+b-1} \cap R_{a+b}=X_{a+b}$ and, as we observed previously, $X_{k}$ is nilpotent for any $k$. In particular, $I$ is a nonzero nilpotent right ideal of $R$, contrary to the semiprimeness of the ring.

The above two results are easily seen to be false without some sort of semiprimeness assumption. Indeed, suppose $R=R_{0} \oplus R_{1}$, where $R_{0}$ is a subring and $R_{1}$ is a nonzero ideal of square 0 . Then this is a finite grading of $R$, and its associated filtration $\mathcal{F}$ satisfies $F_{-1}=0, F_{0}=R_{0}$, and $F_{1}=R$. But $\mathcal{F}$ is not maximal since its 0 -term can certainly be extended to all of $R$. Furthermore, if $R_{0}$ is semiprime and if $\mathcal{G}$ is the filtration of $R$ given by $G_{-1}=0$ and $G_{0}=R$, then $\mathcal{G}$ is not contained in a maximal bounded filtration. Indeed, it is easy to see that any bounded filtration of $R$ containing $\mathcal{G}$ can be extended to a filtration having arbitrarily many negative terms equal to the ideal $R_{1}$.

The problem of characterizing the maximal bounded filtrations of semisimple Artinian rings is surely of interest in its own right. The $*$ version of the problem is perhaps less so. However, it actually arose as a tool in the study of the analogous problem for Lie algebras. Indeed, suppose $L$ is a Lie algebra over a field $K$. A $\mathbb{Z}$-filtration $\mathcal{L}=\left\{L_{i} \mid i \in \mathbb{Z}\right\}$ of $L$ is a collection of $K$-subspaces

$$
\cdots \subseteq L_{-2} \subseteq L_{-1} \subseteq L_{0} \subseteq L_{1} \subseteq L_{2} \subseteq \cdots
$$

indexed by the integers $\mathbb{Z}$ such that $\left[L_{i}, L_{j}\right] \subseteq L_{i+j}$ for all $i, j \in \mathbb{Z}$. The filtration is bounded if there exist integers $\ell$ and $\ell^{\prime}$ with $L_{\ell}=0$ and $L_{\ell^{\prime}}=L$.

In a follow-up paper $\overline{B P}$, Y. Barnea and I study the maximal bounded filtrations of finite-dimensional semisimple complex Lie algebras. The problem quickly reduces to the simple case, so $L$ is of type $\mathrm{A}_{n}, \mathrm{~B}_{n}, \mathrm{C}_{n}, \mathrm{D}_{n}, \mathrm{E}_{6}, \mathrm{E}_{7}, \mathrm{E}_{8}, \mathrm{~F}_{4}$ or $\mathrm{G}_{2}$. As in the case of semisimple rings, the proof proceeds in two steps. The first step is to show that $L_{0}$ contains a Cartan subalgebra $H$ of $L$, and the second step is to classify the maximal bounded filtrations with $L_{0}$ containing a fixed $H$.

Surprisingly, we were not able to achieve the first step by Lie techniques alone. Rather, if $L$ is of type $\mathrm{A}_{n}, \mathrm{~B}_{n}, \mathrm{C}_{n}$, or $\mathrm{D}_{n}$, then $L$ is naturally embedded in a full matrix ring $R=\mathrm{M}_{m}(K)$ over the complex numbers, and we show that the maximal bounded filtration $\mathcal{L}$ extends to a bounded filtration $\mathcal{F}$ of $R$ by defining

$$
F_{a}=\sum L_{i_{1}} L_{i_{2}} \cdots L_{i_{k}}
$$

where the sum is over all subscripts with $i_{1}+i_{2}+\cdots+i_{k} \leq a$. Furthermore, if $\mathcal{G}=\left\{G_{i} \mid i \in \mathbb{Z}\right\}$ is a maximal filtration of $R$ larger than $\mathcal{F}$, then it is easy to see that $G_{i} \cap L=L_{i}$. In particular, if $L$ is of type $\mathrm{A}_{n}$, then the existence of a full diagonal in $G_{0}$ implies that $L_{0}$ does indeed contain a Cartan subalgebra. On the other hand, if $L$ is of type $\mathrm{B}_{n}, \mathrm{C}_{n}$, or $\mathrm{D}_{n}$, then $R$ admits an involution $*$ of the first kind with $L$ being the space of skew-symmetric elements of $R$. In this case, $\mathcal{F}$ is a *-filtration and if we take $\mathcal{G}$ to be $*$-maximal, then Lemma 5.7 yields the result.

\section{ANNIHILATORS AND MAXIMAl FILTRATIONS}

We recall the basic definitions. If $R$ is a ring with 1 , then a $\mathbb{Z}$-filtration $\mathcal{F}=$ $\left\{F_{i} \mid i \in \mathbb{Z}\right\}$ of $R$ is a collection of additive subgroups

$$
\cdots \subseteq F_{-2} \subseteq F_{-1} \subseteq F_{0} \subseteq F_{1} \subseteq F_{2} \subseteq \cdots
$$


indexed by the integers $\mathbb{Z}$ such that $F_{i} F_{j} \subseteq F_{i+j}$ for all $i, j \in \mathbb{Z}$. Furthermore, $\bigcup_{i} F_{i}=R$ and $\bigcap_{i} F_{i}=0$. We say that the filtration is bounded if there exist integers $\ell$ and $\ell^{\prime}$ with $F_{\ell}=0$ and $F_{\ell^{\prime}}=R$. If $\mathcal{G}=\left\{G_{i} \mid i \in \mathbb{Z}\right\}$ is a second such filtration, we say that $\mathcal{G}$ contains $\mathcal{F}$, or $\mathcal{G}$ is larger than $\mathcal{F}$, if $G_{i} \supseteq F_{i}$ for all $i$. In particular, it makes sense to speak about maximal bounded filtrations. The first goal of this paper is to classify the maximal bounded filtrations of Artinian semisimple rings. In this section, we show that if $\mathcal{F}$ is such a maximal filtration, then $F_{0}$ contains a full diagonal of $R$.

Lemma 2.1. Let $\mathcal{F}=\left\{F_{i} \mid i \in \mathbb{Z}\right\}$ be a maximal bounded filtration of $R$. If $C$ is a central subring of $R$, then $C \subseteq F_{0}$ and each $F_{i}$ is a $C$-submodule of $R$.

Proof. If we define $G_{i}=C F_{i}$ for $i<0$ and $G_{i}=C F_{i}+C$ for $i \geq 0$, then it is trivial to check that $\mathcal{G}=\left\{G_{i} \mid i \in \mathbb{Z}\right\}$ is a bounded filtration containing $\mathcal{F}$. In particular, since $\mathcal{F}$ is maximal, we must have $\mathcal{F}=\mathcal{G}$.

Suppose $R=R_{1} \oplus R_{2} \oplus \cdots \oplus R_{k}$ is a finite direct sum of rings and let $\mathcal{F}_{j}=$ $\left\{F_{i j} \mid i \in \mathbb{Z}\right\}$ be a bounded filtration of $R_{j}$ for $j=1,2, \ldots, k$. If we define $F_{i}=$ $F_{i 1} \oplus F_{i 2} \oplus \cdots \oplus F_{i k} \subseteq R$, then $\mathcal{F}=\left\{F_{i} \mid i \in \mathbb{Z}\right\}$ is easily seen to be a bounded filtration of $R$. For convenience, we write $\mathcal{F}=\mathcal{F}_{1} \oplus \mathcal{F}_{2} \oplus \cdots \oplus \mathcal{F}_{k}$ and say that $\mathcal{F}$ is the sum of the various $\mathcal{F}_{j}$ s. With this notation, we have

Lemma 2.2. Let $R=R_{1} \oplus R_{2} \oplus \cdots \oplus R_{k}$ be a finite direct sum of rings and let $\mathcal{F}$ be a bounded $\mathbb{Z}$-filtration of $R$. Then $\mathcal{F}$ is maximal if and only if it is a sum $\mathcal{F}=\mathcal{F}_{1} \oplus \mathcal{F}_{2} \oplus \cdots \oplus \mathcal{F}_{k}$ with each $\mathcal{F}_{j}$ a maximal filtration of $R_{j}$.

Proof. Let $1=e_{1}+e_{2}+\cdots+e_{k}$ be the decomposition of $1 \in R$ into orthogonal central idempotents corresponding to the direct sum $R=R_{1} \oplus R_{2} \oplus \cdots \oplus R_{k}$. If $\mathcal{F}=\left\{F_{i} \mid i \in \mathbb{Z}\right\}$ is a maximal filtration of $R$, then the previous lemma implies that each $e_{j}$ is contained in $F_{0}$. Thus, for each $i$, we have the decomposition $F_{i}=e_{1} F_{i} \oplus e_{2} F_{i} \oplus \cdots \oplus e_{k} F_{i}$ and therefore $\mathcal{F}=\mathcal{F}_{1} \oplus \mathcal{F}_{2} \oplus \cdots \oplus \mathcal{F}_{k}$ where $\mathcal{F}_{j}=$ $\left\{e_{j} F_{i} \mid i \in \mathbb{Z}\right\}$ is a bounded filtration of $R_{j}$. Obviously, each $\mathcal{F}_{j}$ must be maximal.

Conversely, suppose $\mathcal{F}=\mathcal{F}_{1} \oplus \mathcal{F}_{2} \oplus \cdots \oplus \mathcal{F}_{k}$ is a sum of maximal filtrations. Since each $\mathcal{F}_{j}=\left\{F_{i j} \mid i \in \mathbb{Z}\right\}$ is maximal, it follows from Lemma 2.1 that $e_{j} \in F_{0 j}$ and hence that $e_{1}, e_{2}, \ldots, e_{k} \in F_{0}=F_{01} \oplus F_{02} \oplus \cdots \oplus F_{0 k}$. In particular, if $\mathcal{G}=$ $\left\{G_{i} \mid i \in \mathbb{Z}\right\}$ contains $\mathcal{F}$, then $e_{1}, e_{2}, \ldots, e_{k} \in F_{0} \subseteq G_{0}$ and hence $\mathcal{G}$ decomposes as above into a sum $\mathcal{G}=\mathcal{G}_{1} \oplus \mathcal{G}_{2} \oplus \cdots \oplus \mathcal{G}_{k}$. Since $\mathcal{G}_{j}$ contains $\mathcal{F}_{j}$, we conclude that $\mathcal{G}_{j}=\mathcal{F}_{j}$ and therefore that $\mathcal{G}=\mathcal{F}$.

If $i \geq 0$, then $1 \in F_{i}$ and hence the left and right annihilators of these subsets satisfy $\mathrm{l} \operatorname{ann}_{R} F_{i}=\mathrm{r}$. $\operatorname{ann}_{R} F_{i}=0$. Thus the following result has nontrivial content only when $i<0$.

Lemma 2.3. If $\mathcal{F}=\left\{F_{i} \mid i \in \mathbb{Z}\right\}$ is a maximal bounded filtration of $R$, then $F_{0}$ contains $F_{i+1} \cdot\left(1 . \operatorname{ann}_{R} F_{i}\right)$ and $\left(\mathrm{r} . \operatorname{ann}_{R} F_{i}\right) \cdot F_{i+1}$ for all $i$.

Proof. Fix $i$ and set $S=F_{i+1} \cdot\left(1 . \operatorname{ann}_{R} F_{i}\right)$. Then

$$
\begin{aligned}
S F_{-1} S & =F_{i+1} \cdot\left(1 \cdot \operatorname{ann}_{R} F_{i}\right)\left(F_{-1} F_{i+1}\right) \cdot\left(l \cdot \operatorname{ann}_{R} F_{i}\right) \\
& \subseteq F_{i+1} \cdot\left(1 \cdot \operatorname{ann}_{R} F_{i}\right) F_{i} \cdot\left(1 \cdot \operatorname{ann}_{R} F_{i}\right)=0
\end{aligned}
$$

since $\left(\mathrm{l} \operatorname{ann}_{R} F_{i}\right) F_{i}=0$. In particular, $S F_{j} S=0$ for all $j<0$. 
Now, for each integer $a$, define

$$
G_{a}=\sum F_{a_{1}} S F_{a_{2}} S \cdots S F_{a_{r}}
$$

where the sum is over all $r \geq 1$ and all subscripts with $a_{1}+a_{2}+\cdots+a_{r} \leq a$. Here, when $r=1$, the summand is $F_{a_{1}}$, so it follows that $G_{a}$ is an additive subgroup of $R$ containing $F_{a}$. Furthermore, it is clear that $G_{a} \subseteq G_{a+1}$ and that $G_{a} G_{b} \subseteq G_{a+b}$, for all $a, b$, so $\mathcal{G}=\left\{G_{a} \mid a \in \mathbb{Z}\right\}$ is a filtration of $R$. When $F_{\ell^{\prime}}=R$ we have $G_{\ell^{\prime}}=R$ and therefore, to show that $\mathcal{G}$ is bounded, we need only show that some $G_{a}=0$. To this end, recall that $F_{\ell}=0$ for some integer $\ell$. Now, if $G_{a} \neq 0$, then $F_{a_{1}} S F_{a_{2}} S \cdots S F_{a_{r}} \neq 0$ for some $r$ and subscripts with $a_{1}+a_{2}+\cdots+a_{r} \leq a$. Since $S F_{j} S=0$ for all negative $j$, we see that $a_{2}, a_{3}, \ldots, a_{r-1} \geq 0$. Furthermore, $F_{a_{1}}, F_{a_{r}} \neq 0$, so $a_{1}, a_{r}>\ell$, Hence $a \geq a_{1}+a_{2}+\cdots+a_{r}>2 \ell$, so $G_{2 \ell}=0$ and $\mathcal{G}$ is indeed bounded.

In particular, since $\mathcal{F}$ is maximal and $\mathcal{G}$ contains $\mathcal{F}$, we conclude that $\mathcal{F}=\mathcal{G}$. Hence $F_{0}=G_{0} \supseteq F_{0} S F_{0} \supseteq S$, using the fact that $1 \in F_{0}$. A similar argument proves that $F_{0} \supseteq T=\left(\mathrm{r} . \operatorname{ann}_{R} F_{i}\right) \cdot F_{i+1}$ since $T F_{j} T=0$ for all $j<0$.

At this point, it is convenient to isolate the following standard idempotent manipulation.

Lemma 2.4. Let $e$ and $f$ be idempotents of a ring $R$ with $e R \subseteq f R$, and set $g=e f$. Then $g$ is an idempotent with $e R=g R$ and $f g=g=g f$. In particular, $f=g+(f-g)$ is a sum of orthogonal idempotents and hence $f R=g R+(f-g) R$ is a direct sum of right ideals. Furthermore, $1-g=(1-f)+(f-g)$ is a sum of orthogonal idempotents, so $R(1-g)=R(1-f)+R(f-g)$ is a sum of left ideals.

Proof. Since $e \in e R \subseteq f R$, we have $f e=e$. It follows that $e g=g$ and $g e=e$, so $g$ is an idempotent of $R$ with $e R=g R$. Since $f g=g=g f$, simple computations yield the remaining observations.

As a consequence of Lemma 2.3, we can now obtain

Lemma 2.5. Let $\mathcal{F}=\left\{F_{i} \mid i \in \mathbb{Z}\right\}$ be a maximal filtration of $R$ and suppose that $R$ is an Artinian semisimple ring. Then $F_{0}$ contains a full diagonal of $R$. More precisely, we can write $1=e_{1}+e_{2}+\cdots+e_{n}$ as an orthogonal sum of primitive idempotents with $e_{i} R e_{i} \subseteq F_{0}$ for all $i=1,2, \ldots, n$.

Proof. Since $\mathcal{F}$ is bounded, there exists $k \geq 1$ with $F_{-k}=0$, and we consider the descending chain of right ideals of $R$ given by $F_{0} R \supseteq F_{-1} R \supseteq \cdots \supseteq F_{-k} R=0$. Since $R$ is Artinian and semisimple, we can define the idempotents $f_{i}$ by $F_{-i} R=$ $f_{i} R$, and note that $f_{0}=1$ since $1 \in F_{0}$. Thus, we have the inclusions

$$
0=f_{k} R \subseteq f_{k-1} R \subseteq \cdots \subseteq f_{2} R \subseteq f_{1} R \subseteq f_{0} R=R .
$$

Using the previous lemma, we modify $f_{1}, f_{2}, \ldots, f_{k}$ in turn, replacing $f_{i}$ by $f_{i}^{\prime}=$ $f_{i} f_{i-1}$. Once this is done, Lemma 2.4 implies that we have $F_{-i+1} R=f_{i-1} R=$ $f_{i} R+\left(f_{i-1}-f_{i}\right) R \supseteq\left(f_{i-1}-f_{i}\right) R$.

Next, observe that l. $\operatorname{ann}_{R} F_{-i}=1 . \operatorname{ann}_{R}\left(F_{-i} R\right)=1 . \operatorname{ann}_{R} f_{i} R=R\left(1-f_{i}\right)$. Thus, by Lemma 2.4 again,

$$
\text { 1. } \operatorname{ann}_{R} F_{-i}=R\left(1-f_{i}\right)=R\left(1-f_{i-1}\right)+R\left(f_{i-1}-f_{i}\right) \supseteq R\left(f_{i-1}-f_{i}\right) .
$$


With this, Lemma 2.3 now yields

$$
\begin{aligned}
F_{0} & \supseteq F_{-i+1} R \cdot\left(\mathrm{l} \operatorname{ann}_{R} F_{-i}\right) \supseteq\left(f_{i-1}-f_{i}\right) R \cdot R\left(f_{i-1}-f_{i}\right) \\
& =\left(f_{i-1}-f_{i}\right) R\left(f_{i-1}-f_{i}\right)
\end{aligned}
$$

for all $i=1,2, \ldots, k$. In particular,

$$
F_{0} \supseteq \sum_{i=1}^{k}\left(f_{i-1}-f_{i}\right) R\left(f_{i-1}-f_{i}\right)
$$

and observe that the elements $f_{i-1}-f_{i}$ are orthogonal idempotents that sum to $\sum_{i=1}^{k}\left(f_{i-1}-f_{i}\right)=f_{0}-f_{k}=1$. The result now follows once we write each $f_{i-1}-f_{i}$ as an orthogonal sum of primitive idempotents of $R$.

\section{MATRIX RINGS AND DIAGONAL FILTRATIONS}

Let $R=\mathrm{M}_{n}(K)$ be the full $n \times n$ matrix ring over the division ring $K$ and fix matrix units $e_{i j}$ for $i, j=1,2, \ldots, n$. For convenience, we say that a bounded $\mathbb{Z}$-filtration $\mathcal{F}=\left\{f_{a} \mid a \in \mathbb{Z}\right\}$ of $R$ is diagonal if $F_{0}$ contains the set of diagonal matrices $\mathrm{D}_{n}(K)=\sum_{i=1}^{n} e_{i i} K$. In this section, we determine the maximal bounded $\mathbb{Z}$-filtrations of $R$ that are also diagonal. Note that, if $\mathcal{G}=\left\{G_{a} \mid a \in \mathbb{Z}\right\}$ is a filtration containing the filtration $\mathcal{F}$, then $G_{0} \supseteq F_{0} \supseteq \mathrm{D}_{n}(K)$ and hence $\mathcal{G}$ is also diagonal. The basic tool here is the degree function $f$ of $\mathcal{F}$ defined by

$$
f(i, j)=\min \left\{a \in \mathbb{Z} \mid e_{i j} \in F_{a}\right\} .
$$

Since $\mathcal{F}$ is bounded, $f(i, j)$ is a well-defined integer for all $i, j=1,2, \ldots, n$.

Lemma 3.1. Let $\mathcal{F}=\left\{F_{a} \mid a \in \mathbb{Z}\right\}$ be a diagonal filtration of $R=\mathrm{M}_{n}(K)$ and let $f$ be its degree function.

(i) For each $a \in \mathbb{Z}$, we have $F_{a}=\sum e_{i j} K$ where the sum is over all $i, j$ with $f(i, j) \leq a$. In particular, $f$ uniquely determines the filtration.

(ii) $f(i, j)+f(j, k) \geq f(i, k)$ for all $i, j, k=1,2, \ldots, n$.

(iii) If $\mathcal{G}=\left\{G_{a} \mid a \in \mathbb{Z}\right\}$ is a diagonal filtration with degree function $g$, then $\mathcal{G}$ contains $\mathcal{F}$ if and only if $g(i, j) \leq f(i, j)$ for all $i, j=1,2, \ldots, n$.

Proof. (i) Since $F_{0} \supseteq \mathrm{D}_{n}(K)$, it follows that

$$
F_{a}=\mathrm{D}_{n}(K) \cdot F_{a} \cdot \mathrm{D}_{n}(K)=\sum_{i, j} F_{a} \cap e_{i j} K
$$

for all $a \in \mathbb{Z}$. Furthermore, since $K \subseteq \mathrm{D}_{n}(K)$ is a division ring and $F_{a}$ is a $K$-module, we see that $F_{a} \cap e_{i j} K=0$ or $e_{i j} K$, and the latter possibility occurs precisely when $f(i, j) \leq a$.

(ii) Say $f(i, j)=a$ and $f(j, k)=b$. Then $e_{i j} \in F_{a}$ and $e_{j k} \in F_{b}$, so $e_{i k}=e_{i j} e_{j k} \in$ $F_{a} F_{b} \subseteq F_{a+b}$. By definition, we have $f(i, k) \leq a+b=f(i, j)+f(j, k)$.

(iii) In view of the above, the inclusions $F_{a} \subseteq G_{a}$ for all $a \in \mathbb{Z}$ occur if and only if $e_{i j} \in F_{a}$ implies that $e_{i j} \in G_{a}$. Furthermore, the latter occurs precisely when $f(i, j) \geq g(i, j)$ for all $i, j=1,2, \ldots, n$.

Let $w$ be a function that associates to each $i=1,2, \ldots, n$ the integer $w_{i}$. Using this weight function $w$, we can define the additive subgroups $R_{a}$ of $R=\mathrm{M}_{n}(K)$ by $R_{a}=\sum e_{i j} K$ where the sum is over all $i, j$ with $w_{i}-w_{j}=a$. It is easy to see that $R$ is the direct sum of these $R_{a}$ s with $a \in \mathbb{Z}$, that almost all $R_{a} \mathrm{~s}$ are 0 , and that $R_{a} R_{b} \subseteq R_{a+b}$. In other words, $R=\sum_{a} R_{a}$ is a finite $\mathbb{Z}$-grading of $R$. Indeed, 
according to $\left[\mathrm{ZS}\right.$, all finite $\mathbb{Z}$-gradings of $\mathrm{M}_{n}(K)$ are essentially of this form. Now any such grading gives rise to a bounded $\mathbb{Z}$-filtration $\mathcal{F}_{w}=\left\{F_{a} \mid a \in \mathbb{Z}\right\}$ by defining

$$
F_{a}=\sum_{b \leq a} R_{b}=\sum e_{i j} K
$$

where the latter sum is over all $i, j=1,2, \ldots, n$ with $w_{i}-w_{j} \leq a$. We call this the weight filtration associated to $w$.

Since the gradings and filtrations above depend upon differences of weight values, it is clear that if we add a constant $c$ to all $w_{i}$ then we obtain the same structure. Thus, we say that two weight functions $v$ and $w$ are equivalent if and only if $v_{i}-w_{i}=v_{j}-w_{j}$ for all $i, j$. With this notation, equivalent weights give rise to the same filtration.

Lemma 3.2. If $w$ is a weight function, then $\mathcal{F}_{w}=\left\{F_{a} \mid a \in \mathbb{Z}\right\}$ is a diagonal $\mathbb{Z}$-filtration with degree function $f_{w}$ given by $f_{w}(i, j)=w_{i}-w_{j}$. Furthermore, if $v$ is a second weight function and if $\mathcal{F}_{v}$ contains $\mathcal{F}_{w}$, then $v$ and $w$ are equivalent, and hence $\mathcal{F}_{v}=\mathcal{F}_{w}$.

Proof. We have already observed that $\mathcal{F}_{w}$ is a bounded $\mathbb{Z}$-filtration. It is diagonal since $w_{i}-w_{i}=0$ implies that $e_{i i} K \subseteq F_{0}$. Furthermore, by definition, $e_{i j} \in F_{a}$ if and only if $w_{i}-w_{j} \leq a$, so $f_{w}(i, j)$ is the minimal such $a$, namely $w_{i}-w_{j}$.

Now let $f_{v}$ be the degree function of $\mathcal{F}_{v}$ so that $f_{v}(i, j)=v_{i}-v_{j}$. If $\mathcal{F}_{v}$ contains $\mathcal{F}_{w}$, then part (iii) of the previous lemma implies that $v_{i}-v_{j}=f_{v}(i, j) \leq f_{w}(i, j)=$ $w_{i}-w_{j}$. In other words, $v_{i}-w_{i} \leq v_{j}-w_{j}$ for all $i, j=1,2, \ldots, n$. But then relabeling yields $v_{j}-w_{j} \leq v_{i}-w_{i}$ for all $i, j$, so $v_{i}-w_{i}=v_{j}-w_{j}$ and hence $v$ and $w$ are equivalent.

If $r$ is a real number, we let $\lceil r\rceil$ denote the smallest integer greater than or equal to $r$. In other words, $\lceil r\rceil \in \mathbb{Z}$ with $\lceil r\rceil \geq r$, and if $m \in \mathbb{Z}$ with $m \geq r$, then $m \geq\lceil r\rceil$. For later applications, it is convenient to isolate the following well-known facts.

Lemma 3.3. If $x$ and $y$ are real numbers, then $\lceil x\rceil \geq x,\lceil x\rceil+\lceil y\rceil \geq x+y$, and $\lceil y-x\rceil \geq\lceil y\rceil-\lceil x\rceil$.

Proof. Note that $\lceil x\rceil+\lceil y\rceil \geq x+y$, and hence $\lceil x\rceil+\lceil y\rceil \geq\lceil x+y\rceil$. Consequently, $\lceil x\rceil+\lceil y-x\rceil \geq\lceil y\rceil$, and therefore $\lceil y-x\rceil \geq\lceil y\rceil-\lceil x\rceil$.

We now come to a key observation.

Lemma 3.4. Let $\mathcal{F}=\left\{F_{a} \mid a \in \mathbb{Z}\right\}$ be a diagonal filtration of $\mathrm{M}_{n}(K)$ with degree function $f$. Then there exists an integer valued weight function $w$ with $f(i, j) \geq$ $w_{i}-w_{j}$ for all $i, j$. In particular, $\mathcal{F}$ is contained in the weight filtration $\mathcal{F}_{w}$.

Proof. Let $f$ be the degree function of $\mathcal{F}$, define the numbers $w_{i}^{\prime}=\frac{1}{n} \sum_{k=1}^{n} f(i, k)$ for all $i=1,2, \ldots, n$, and set $w_{i}=\left\lceil w_{i}^{\prime}\right\rceil \in \mathbb{Z}$. We claim that the weight filtration $\mathcal{F}_{w}$ contains $\mathcal{F}$. To this end, observe that $f(i, j) \geq f(i, k)-f(j, k)$ for all $i, j, k=$ $1,2, \ldots, n$, by Lemma 3.1(ii). Hence, if we sum this inequality over all $k=1,2, \ldots, n$ and divide by $n$, we obtain

$$
f(i, j) \geq \frac{1}{n} \sum_{k=1}^{n} f(i, k)-\frac{1}{n} \sum_{k=1}^{n} f(j, k)=w_{i}^{\prime}-w_{j}^{\prime} .
$$

But, $f(i, j) \in \mathbb{Z}$, so the previous two lemmas yield

$$
f(i, j) \geq\left\lceil w_{i}^{\prime}-w_{j}^{\prime}\right\rceil \geq\left\lceil w_{i}^{\prime}\right\rceil-\left\lceil w_{j}^{\prime}\right\rceil=w_{i}-w_{j}=f_{w}(i, j),
$$


where $f_{w}$ is the degree function of $\mathcal{F}_{w}$. Since both $\mathcal{F}$ and $\mathcal{F}_{w}$ are diagonal, Lemma 3.1(iii) and the inequalities $f(i, j) \geq f_{w}(i, j)$ for all $i, j=1,2, \ldots, n$ imply that $\mathcal{F}_{w}$ is indeed larger than $\mathcal{F}$.

With this, we have

Lemma 3.5. Let $R=\mathrm{M}_{n}(K)$ be the full $n \times n$ matrix ring over the division ring $K$. A diagonal filtration of $R$ is maximal if and only if it is a weight filtration. Furthermore, the weight filtrations for nonequivalent weights are all distinct.

Proof. If $\mathcal{F}$ is a maximal diagonal filtration, then the preceding lemma implies that $\mathcal{F}=\mathcal{F}_{w}$ is a weight filtration.

Conversely, suppose that $\mathcal{F}_{w}$ is any weight filtration and let $\mathcal{F}$ contain $\mathcal{F}_{w}$. Then $\mathcal{F}$ is a diagonal filtration, so the preceding lemma again applies and we conclude that there is a weight filtration $\mathcal{F}_{v}$ containing $\mathcal{F}$ and hence containing $\mathcal{F}_{w}$. But then, Lemma 3.2 implies that $v$ and $w$ are equivalent weights and hence that $\mathcal{F}_{v}=\mathcal{F}=\mathcal{F}_{w}$. Consequently, $\mathcal{F}_{w}$ is maximal.

It is now a simple matter to combine all of these ingredients.

Theorem 3.6. Let $R$ be a semisimple Artinian ring and let $\mathcal{F}=\left\{F_{i} \mid i \in \mathbb{Z}\right\}$ be a bounded $\mathbb{Z}$-filtration of $R$.

(i) If we write $R=R_{1} \oplus R_{2} \oplus \cdots \oplus R_{k}$ as a finite direct sum of simple Artinian rings, then $\mathcal{F}$ is maximal if and only if it is a sum $\mathcal{F}=\mathcal{F}_{1} \oplus \mathcal{F}_{2} \oplus \cdots \oplus \mathcal{F}_{k}$, where each $\mathcal{F}_{j}$ is a maximal filtration of $R_{j}$.

(ii) If $R$ is simple, then $\mathcal{F}$ is maximal if and only if there exists a suitable description of $R$ as a full matrix ring over a division ring such that $\mathcal{F}$ becomes a weight filtration of $R$.

Proof. (i) This is essentially a restatement of Lemma 3.2

(ii) Now suppose that $R$ is simple and $\mathcal{F}$ is maximal. By Lemma 2.5, $F_{0}$ contains a full diagonal of $R$. Specifically, we can write $1=e_{1}+e_{2}+\cdots+e_{n}$ as an orthogonal sum of primitive idempotents with $e_{i} R e_{i} \subseteq F_{0}$ for all $i=1,2, \ldots, n$. Then, using these idempotents, we can write $R$ as a full matrix ring $\mathrm{M}_{n}(K)$ over a division ring $K$, such that $e_{i i} R e_{i i} \subseteq F_{0}$ for all $i=1,2, \ldots, n$. With this, Lemma 3.5 implies that $\mathcal{F}$ is a weight filtration. Conversely, if $\mathcal{F}$ is a weight filtration, then we conclude from Lemma 3.5 again that $\mathcal{F}$ is maximal.

\section{MATRIX RINGS With INVOLUTION}

The results of the next two sections will be needed in the later paper BP on filtrations in semisimple Lie algebras. Specifically, our concern here is with matrix rings admitting an involutary antiautomorphism *. We begin with certain more general considerations.

Lemma 4.1. Let $R$ be a ring with involution $*$ and assume that $1 / 2 \in R$. Suppose $e \in R$ is an idempotent with $e^{*} e=0$. If $f=e\left(1-e^{*} / 2\right)$, then $f$ is an idempotent in $R$ with $e f=f$ and $f e=e$, so that $e R=f R$. Furthermore, $f^{*} f=0=f f^{*}$.

Proof. It is clear that $e f=f$, and obviously $f e=e$ follows from $e^{*} e=0$. Furthermore, $e^{*} e=0$ implies that $f^{*} f=0$, and then $f f^{*}=e\left(1-e^{*} / 2\right)(1-e / 2) e^{*}=$ $e\left(1-e / 2-e^{*} / 2\right) e^{*}=0$. 
Of course, the true function of the fraction $1 / 2$ above is that it satifies the equation $(1 / 2)+(1 / 2)^{*}=1$. The following is a variant of an argument due to I. Kaplansky in the proof of $[\mathrm{H}$, Theorem 1.2.2]. While we will need it only in the case of matrix rings over division rings, the generalization to semiprime rings is no harder to prove.

Lemma 4.2. Let $R$ be a semiprime ring with involution * and let $I$ and $J$ be minimal right ideals of $R$.

(i) If $J^{*} I \neq 0$, there exists an idempotent $e \in I$ with $I=e R$ and $J=e^{*} R$.

(ii) If $I^{*} I \neq 0$, there exists a *-symmetric idempotent $e \in I$ with $I=e R$.

Proof. (i) By assumption, there exists $y \in J$ with $y^{*} I \neq 0$. Since $R$ is semiprime, we have $y^{*} I y^{*} I \neq 0$ and hence there exists $x \in I$ with $x y^{*} I \neq 0$. Now $I_{R}$ is minimal and $0 \neq x y^{*} I \subseteq I$, so $x y^{*} I=I$. In particular, there exists $e \in I$ with $x y^{*} e=x y^{*}$, and hence $x y^{*}\left(e^{2}-e\right)=0$. But then $e^{2}-e \in \mathrm{r} \cdot \operatorname{ann}_{I}\left(x y^{*}\right)$ and the latter is a right ideal of $R$ properly smaller than $I$. Thus r. $\operatorname{ann}_{I}\left(x y^{*}\right)=0$, so $e=e^{2}$ is an idempotent. Obviously $e \neq 0$, since $x y^{*} e=x y^{*}$, and thus $I=e R$.

Next, applying $*$ to $x y^{*} e=x y^{*} \neq 0$ yields $e^{*} y x^{*}=y x^{*} \neq 0$, and hence $e^{*} J=$ $e^{*} y x^{*} R=y x^{*} R=J$ since $J_{R}$ is minimal. Furthermore, since $R$ is semiprime, and $e R$ is a minimal right ideal of $R,[\mathrm{H}$, Lemma 1.2.1] implies that $R e$ is a minimal left ideal of $R$ and hence that $e^{*} R=(R e)^{*}$ is minimal on the right. Since $e^{*} R \supseteq$ $e^{*} J=J$, it follows that $e^{*} R=J$, as required.

(ii) By the above, there exists an idempotent $e \in I$ with $I=e R=e^{*} R$. Since $e^{*}$ is a left identity for $I$, we conclude that $e=e^{*} e$ is $*$-symmetric.

Now let $K$ be a division ring of characteristic $\neq 2$ and let $R=\mathrm{M}_{n}(K)$. Furthermore, let $U=\mathrm{U}_{n}(K)$ denote the $K$-subalgebra of $R$ consisting of all the upper triangular matrices. We assume that $R$ admits an involution $*$.

Lemma 4.3. With the above notation, there exists a primitive idempotent $f \in$ $e_{11} R \subseteq U$ with $f^{*} \in U$. Furthermore, either $f=f^{*}$ or $f f^{*}=0=f^{*} f$.

Proof. Let $I=e_{11} R$ and, for each $i$, set $J_{i}=e_{i i} R$. Then these are minimal right ideals of $R$. Furthermore, since $\sum_{i=1}^{n} J_{i}=R$, it follows that $J_{i}^{*} I \neq 0$ for some $i$, and we let $k$ be the minimal subscript with $J_{k}^{*} I \neq 0$. If $k=1$, then $I^{*} I \neq 0$ and Lemma 4.2 (ii) implies that $I$ contains a $*$-symmetric primitive idempotent, as required.

On the other hand, if $k>1$, then Lemma 4.2(i) at least implies that $I$ contains a primitive idempotent $e$ with $e^{*} \in J_{k}$. In other words, $e^{*}$ has nonzero entries only in the $k$ th row of the matrix ring. Since $k>1$, we have $I^{*} I=0$ and hence $e^{*} e=0$. Furthermore, by the minimality of $k$, we have $J_{i}^{*} I=0$ for $i=1,2, \ldots, k-1$, so $e_{i i}^{*} e=0$ for these values of $i$. Hence $0=\left(e_{i i}^{*} e\right)^{*}=e^{*} e_{i i}$, and this shows that $e^{*}$ has only zero entries in columns $i=1,2, \ldots, k-1$. Since $e^{*} \in J_{k}$, we conclude that $e^{*} \in U$. Finally, if we set $f=e\left(1-e^{*} / 2\right) \in I \subseteq U$, then Lemma 4.1 implies that $f$ is an idempotent with $e f=f, f e=e$ and $f^{*} f=0=f f^{*}$. Since $f^{*}=(1-e / 2) e^{*} \in U$, the result follows.

With this in hand, we can now prove the following somewhat surprising proposition.

Proposition 4.4. Let $R=\mathrm{M}_{n}(K)$ be a full matrix ring over a division ring $K$ of characteristic $\neq 2$, and let $U=\mathrm{U}_{n}(K)$ be its $K$-subalgebra of upper triangular 
matrices. If $R$ admits an involution $*$, then we can write $1=e_{1}+e_{2}+\cdots+e_{n}$ as a sum of orthogonal primitive idempotents in $U$ with the set $\left\{e_{1}, e_{2}, \ldots, e_{n}\right\}$ being stable under the action of $*$.

Proof. We proceed by induction on $n$, the case $n=1$ being trivial. Now suppose that $n \geq 2$. By the previous lemma, $e_{11} R \subseteq U$ contains a primitive idempotent $f$ with $f^{*} \in U$ and with either $f=f^{*}$ or $f f^{*}=0=f^{*} f$. Suppose first that $f=f^{*}$ and let $1=f_{1}+f_{2}+\cdots+f_{n}$ be an orthogonal decomposition of 1 in $U$ with $f=f_{1}$. Since $1=e_{11}+e_{22}+\cdots+e_{n n}$ is a second such decomposition in $U$, there exists a unit $u \in U$ with $u^{-1} f_{i} u=e_{i i}$. Note that $e_{11}$ corresponds to $f=f_{1}$ because these idempotents are identical modulo the radical of $U$. Since $u^{-1} U u=U$, we can now assume without loss of generality that $f=e_{11}$. Now $f$ is $*$-stable, so $*$ acts on $(1-f) R(1-f)=\left(1-e_{11}\right) R\left(1-e_{11}\right) \cong \mathrm{M}_{n-1}(K)$. Furthermore, under this isomorphism, $(1-f) U(1-f)$ corresponds to $\mathrm{U}_{n-1}(K)$. Thus, by induction, $1-f=e_{2}+e_{3}+\cdots+e_{n}$ is an orthogonal sum of primitive idempotents in $(1-f) U(1-f) \subseteq U$ with $\left\{e_{2}, e_{3}, \ldots, e_{n}\right\}$ a $*$-stable set. The result now follows in this case by setting $e_{1}=f$.

The case where $f f^{*}=0=f^{*} f$ is similar. We first extend $\left\{f, f^{*}\right\}$ to a maximal set $\left\{f_{1}, f_{2}, \ldots, f_{n}\right\}$ of orthogonal primitive idempotents, with labels chosen so that there exists a unit $u$ in $U$ with $u^{-1} f_{i} u=e_{i i}$. Obviously, $f_{1}=f$ and $f_{k}=f^{*}$ for some $k>1$. Since $u^{-1} U u=U$, we can now assume without loss of generality that $f=e_{11}$ and $f^{*}=e_{k k}$. Note that $f+f^{*}$ is a $*$-stable idempotent, so $*$ acts on $\left(1-f-f^{*}\right) R\left(1-f-f^{*}\right)=\left(1-e_{11}-e_{k k}\right) R\left(1-e_{11}-e_{k k}\right) \cong \mathrm{M}_{n-2}(K)$. Furthermore, under this isomorphism, $\left(1-f-f^{*}\right) U\left(1-f-f^{*}\right)$ corresponds to $\mathrm{U}_{n-2}(K)$. Thus, by induction if $n>2$, or trivially if $n=2$, we can write $1-f-f^{*}=e_{3}+e_{4}+\cdots+e_{n}$ as an orthogonal sum of primitive idempotents in $\left(1-f-f^{*}\right) U\left(1-f-f^{*}\right) \subseteq U$ with $\left\{e_{3}, e_{4} \ldots, e_{n}\right\}$ a $*$-stable set. The result now follows in this case by setting $e_{1}=f$ and $e_{2}=f^{*}$.

Again, suppose that $R=\mathrm{M}_{n}(K)$ is the full ring of $n \times n$ matrices over the division ring $K$ of characteristic $\neq 2$. We study the $*$-filtrations of $R$. These are ordinary $\mathbb{Z}$-filtrations $\mathcal{F}=\left\{F_{i} \mid i \in \mathbb{Z}\right\}$ but with the additional assumption that $F_{i}^{*}=F_{i}$ for all $i$. In other words, each $F_{i}$ is $*$-stable. Again, we speak of bounded $*$-filtrations and then of $*$-filtrations maximal in the collection of bounded $*$-filtrations. For the sake of clarity, we say that the latter filtrations are $*$-maximal. The key application of the above work is

Lemma 4.5. Let $\mathcal{F}=\left\{F_{i} \mid i \in \mathbb{Z}\right\}$ be a*-maximal bounded $*$-filtration of $R$. Then $F_{0}$ contains a $*$-stable full diagonal. Specifically, we can write $1=e_{1}+e_{2}+\cdots+e_{n}$ as an orthogonal sum of primitive idempotents with $\mathrm{D}_{n}(K)=\sum_{i=1}^{n} K e_{i} \subseteq F_{0}$ and with $\left\{e_{1}, e_{2}, \ldots, e_{n}\right\}$ being a*-stable set.

Proof. Let $\mathcal{F} \subseteq \mathcal{G}$ where $\mathcal{G}=\left\{G_{i} \mid i \in \mathbb{Z}\right\}$ is a maximal bounded ordinary $\mathbb{Z}$ filtration of $R$. Then $\mathcal{G}$ satisfies the conclusion of Theorem 3.6(ii). In particular, there exists a suitable description of $R$ as a full matrix ring over $K$ such that $\mathcal{G}$ becomes a weight filtration. We can, of course, label the $e_{i j}$ subscripts so that the weights satisfy $w_{1} \leq w_{2} \leq \cdots \leq w_{n}$. Then $i \leq j$ implies that $w_{i}-w_{j} \leq 0$ and hence that $K e_{i j} \subseteq G_{0}$. In other words, $G_{0}$ contains $U=\mathrm{U}_{n}(K)$, the space of upper triangular matrices, and Proposition 4.4 implies that $G_{0}$ contains $\left\{e_{1}, e_{2}, \ldots, e_{n}\right\}$ a *-stable set of primitive orthogonal idempotents of $U$ which sum to 1 . Indeed, $G_{0}$ contains $\mathrm{D}_{n}(K)=\sum_{i=1}^{n} R e_{i} R=\sum_{i=1}^{n} K e_{i} \subseteq U$, and this set is clearly *-stable. 
Note that $\bar{G}_{i}=G_{i} \cap G_{i}^{*}$ is a $*$-stable additive subgroup of $R$ that contains $F_{i}$. Furthermore, $\bar{G}_{i} \bar{G}_{j} \subseteq G_{i} G_{j} \subseteq G_{i+j}$ and $\bar{G}_{i} \bar{G}_{j} \subseteq G_{i}^{*} G_{j}^{*}=\left(G_{j} G_{i}\right)^{*} \subseteq G_{j+i}^{*}=G_{i+j}^{*}$. Thus $\bar{G}_{i} \bar{G}_{j} \subseteq \bar{G}_{i+j}$, and hence $\overline{\mathcal{G}}=\left\{\bar{G}_{i} \mid i \in \mathbb{Z}\right\}$ is a bounded $*$-filtration containing $\mathcal{F}$. But $\mathcal{F}$ is $*$-maximal, so $\mathcal{F}=\overline{\mathcal{G}}$. In particular, $F_{0}=\bar{G}_{0} \supseteq \mathrm{D}_{n}(K)$, as described above. This completes the proof.

\section{Diagonal Filtrations in matriX RINGS With INVOLUtion}

Let $R=\mathrm{M}_{n}(K)$ be the full $n \times n$ matrix ring over the division ring $K$ and let * be an involution defined on $R$. Fix matrix units $e_{i j}$ for $i, j=1,2, \ldots, n$ and assume that the set $\left\{e_{11}, e_{22}, \ldots, e_{n n}\right\}$ is $*$-stable. We can then define a corresponding map $*$ on the subscripts $\{1,2, \ldots, n\}$ by setting $e_{i i}^{*}=e_{i^{*} i^{*}}$. Obviously, $*$ has order at most 2 as a permutation.

As usual, we say that a bounded $\mathbb{Z}$-filtration $\mathcal{F}=\left\{F_{a} \mid a \in \mathbb{Z}\right\}$ of $R$ is diagonal if $F_{0}$ contains the set of diagonal matrices $\mathrm{D}_{n}(K)=\sum_{i=1}^{n} e_{i i} K$. In this section, we determine the $*$-maximal bounded $*$-filtrations of $R$ that are also diagonal. Note that, if $\mathcal{G}=\left\{G_{a} \mid a \in \mathbb{Z}\right\}$ is a $*$-filtration containing the filtration $\mathcal{F}$, then $G_{0} \supseteq F_{0} \supseteq \mathrm{D}_{n}(K)$ and hence $\mathcal{G}$ is also diagonal. The argument here again makes use of the degree function $f$ of $\mathcal{F}$ defined by

$$
f(i, j)=\min \left\{a \in \mathbb{Z} \mid e_{i j} \in F_{a}\right\} .
$$

Since $\mathcal{F}$ is bounded, $f(i, j)$ is a well-defined integer for all $i, j=1,2, \ldots, n$.

Lemma 5.1. Let $\mathcal{F}=\left\{F_{a} \mid a \in \mathbb{Z}\right\}$ be a diagonal filtration of $R=\mathrm{M}_{n}(K)$ and let $f$ be its degree function.

(i) For each $a \in \mathbb{Z}$, we have $F_{a}=\sum e_{i j} K$ where the sum is over all $i, j$ with $f(i, j) \leq a$. In particular, $f$ uniquely determines the filtration.

(ii) If $\mathcal{G}=\left\{G_{a} \mid a \in \mathbb{Z}\right\}$ is a second diagonal filtration with degree function $g$, then $\mathcal{G}$ contains $\mathcal{F}$ if and only if $g(i, j) \leq f(i, j)$ for all $i, j=1,2, \ldots, n$.

(iii) $\mathcal{F}$ is $a *$-filtration if and only if $f(i, j)=f\left(j^{*}, i^{*}\right)$ for all $i, j=1,2, \ldots, n$.

Proof. Since $\mathcal{F}$ is an ordinary filtration, parts (i) and (ii) follow from Lemma 3.1. For (iii), notice that $e_{i j} K=e_{i i} R e_{j j}$ and hence $\left(e_{i j} K\right)^{*}=e_{j j}^{*} R e_{i i}^{*}=e_{j^{*} j^{*}} R e_{i^{*} i^{*}}=$ $e_{j^{*} i^{*}} K$. Thus $\mathcal{F}$ is a $*$-filtration if and only if $e_{i j} K$ and $e_{j^{*} i^{*}} K$ are in the same $F_{a}$ terms and hence if and only if $f(i, j)=f\left(j^{*}, i^{*}\right)$.

Let $w$ be a function that associates to each $i=1,2, \ldots, n$ an integer or a half integer $w_{i}$ and assume that $w_{i}+w_{i^{*}}=0$ for all $i$. Using this $*$-weight function $w$, we define a filtration $\mathcal{F}_{w}$ on $R=\mathrm{M}_{n}(K)$ by setting $F_{a}=\sum e_{i j} K$, where the latter sum is over all $i, j=1,2, \ldots, n$ with $w_{i}-w_{j} \leq a$. We call this the $*$-weight filtration associated to $w$.

Lemma 5.2. If $w$ is a $*$-weight function, then $\mathcal{F}_{w}=\left\{F_{a} \mid a \in \mathbb{Z}\right\}$ is a diagonal *-filtration with degree function $f_{w}$ given by $f_{w}(i, j)=\left\lceil w_{i}-w_{j}\right\rceil$. Furthermore, if $v$ is a second $*$-weight function and if $\mathcal{F}_{v}$ contains $\mathcal{F}_{w}$, then $v$ and $w$ are equal and hence $\mathcal{F}_{v}=\mathcal{F}_{w}$.

Proof. If $e_{i j} K \subseteq F_{a}$ and $e_{j k} K \subseteq F_{b}$, then $a \geq w_{i}-w_{j}$ and $b \geq w_{j}-w_{k}$. Thus $a+b \geq w_{i}-w_{k}$, so $\left(e_{i j} K\right)\left(e_{j k} K\right)=e_{i k} K \subseteq F_{a+b}$, and it follows that $\mathcal{F}_{w}$ is indeed a filtration. Furthermore, it is diagonal since $w_{i}-w_{i}=0$ implies that $e_{i i} K \subseteq F_{0}$ for all $i$, and its degree function is clearly $f_{w}(i, j)=\left\lceil w_{i}-w_{j}\right\rceil$. Since $w_{i^{*}}=-w_{i}$, we have $f_{w}(i, j)=f_{w}\left(j^{*}, i^{*}\right)$, and hence $\mathcal{F}_{w}$ is a $*$-filtration. 
Now let $f_{v}$ be the degree function of the $*$-weight filtration $\mathcal{F}_{v}$ so that $f_{v}(i, j)=$ $\left\lceil v_{i}-v_{j}\right\rceil$. If $\mathcal{F}_{v}$ contains $\mathcal{F}_{w}$, then part (ii) of the previous lemma implies that $\left\lceil v_{i}-v_{j}\right\rceil=f_{v}(i, j) \leq f_{w}(i, j)=\left\lceil w_{i}-w_{j}\right\rceil$. In particular, since $\left\lceil v_{i}-v_{i^{*}}\right\rceil=$ $\left\lceil v_{i}+v_{i}\right\rceil=\left\lceil 2 v_{i}\right\rceil=2 v_{i}$, we conclude that $v_{i} \leq w_{i}$ for all $i$. Replacing $i$ by $i^{*}$ then yields $-v_{i}=v_{i^{*}} \leq w_{i^{*}}=-w_{i}$, so $w_{i} \leq v_{i}$ and hence $v=w$, as required.

Next comes a key observation.

Lemma 5.3. If $\mathcal{F}=\left\{F_{a} \mid a \in \mathbb{Z}\right\}$ is a diagonal $*$-filtration of $R=\mathrm{M}_{n}(K)$, then $\mathcal{F}$ is contained in a $*$-weight filtration.

Proof. Let $f$ be the degree function of $\mathcal{F}$. In view of Lemma 3.4, there exists an integer valued function $v$ with $f(i, j) \geq v_{i}-v_{j}$ for all $i, j$. Furthermore, $f(i, j)=$ $f\left(j^{*}, i^{*}\right) \geq v_{j^{*}}-v_{i^{*}}$. Thus, if we set $w_{i}=\left(v_{i}-v_{i^{*}}\right) / 2$, then it is clear that $w$ is a *-weight function with values in $\mathbb{Z} / 2$ and with $f(i, j) \geq w_{i}-w_{j}$. We conclude that $f(i, j) \geq\left\lceil w_{i}-w_{j}\right\rceil=f_{w}(i, j)$, and hence that $\mathcal{F} \subseteq \mathcal{F}_{w}$.

With this, we obtain

Lemma 5.4. Let $R=\mathrm{M}_{n}(K)$ be an $n \times n$ matrix ring over a division ring $K$. A diagonal $*$-filtration of $R$ is $*$-maximal if and only if it is a $*$-weight filtration. Furthermore, the $*$-weight filtrations for distinct $*$-weights are all different.

Proof. If $\mathcal{F}$ is a $*$-maximal diagonal $*$-filtration, then the preceding lemma implies that $\mathcal{F}=\mathcal{F}_{w}$ is a $*$-weight filtration.

Conversely, suppose that $\mathcal{F}_{w}$ is any $*$-weight filtration and let $\mathcal{F}$ be a $*$-filtration containing $\mathcal{F}_{w}$. Then $\mathcal{F}$ is a diagonal $*$-filtration, so the preceding lemma again applies and we conclude that there is a $*$-weight filtration $\mathcal{F}_{v}$ containing $\mathcal{F}$ and hence containing $\mathcal{F}_{w}$. But then, Lemma 5.2 implies that $v$ and $w$ are equal, so $\mathcal{F}_{v}=\mathcal{F}=\mathcal{F}_{w}$ and $\mathcal{F}_{w}$ is indeed $*$-maximal.

It is now a simple matter to combine all of these ingredients. The following is our main result on $*$-filtrations.

Theorem 5.5. Let $R$ be a simple Artinian ring of characteristic $\neq 2$ admitting an involution $*$, and let $\mathcal{F}=\left\{F_{i} \mid i \in \mathbb{Z}\right\}$ be a bounded $*$-filtration of $R$. Then $\mathcal{F}$ is *-maximal if and only if there exists a suitable description of $R$ as a full $n \times n$ matrix ring over a division ring with $\left\{e_{11}, e_{22}, \ldots, e_{n n}\right\}$ stable under the action of * and with $\mathcal{F}=\mathcal{F}_{w}$ being a $*$-weight filtration of $R$.

Proof. Suppose first that $\mathcal{F}$ is $*$-maximal. By Lemma 4.5 $F_{0}$ contains a full diagonal of $R$ compatible with $*$. Specifically, we can write $1=e_{1}+e_{2}+\cdots+e_{n}$ as an orthogonal sum of primitive idempotents with $e_{i} R e_{i} \subseteq F_{0}$ for all $i=1,2, \ldots, n$, and with $\left\{e_{1}, e_{2}, \ldots, e_{n}\right\}$ stable under $*$. Then, using these idempotents, we can write $R$ as a full matrix ring $\mathrm{M}_{n}(K)$ over a division ring $K$, such that $e_{i i} K=e_{i i} R e_{i i} \subseteq F_{0}$ for all $i=1,2, \ldots, n$. With this, Lemma 5.4 implies that $\mathcal{F}$ is a $*$-weight filtration. Conversely, if $\mathcal{F}$ is a $*$-weight filtration, then we conclude from Lemma 5.4 again that $\mathcal{F}$ is $*$-maximal.

It is interesting to see which $*$-maximal filtrations are maximal as ordinary filtrations. In the following, when we speak about the parity of a number in $\mathbb{Z} / 2$, we will mean the parity of its numerator. Thus, the even parity numbers are precisely the integers, while the odd parity numbers are elements of the set $\mathbb{Z}+(1 / 2)$. 
Lemma 5.6. Let $\mathcal{F}_{w}$ be a $*$-weight filtration. Then $\mathcal{F}_{w}$ is maximal as an ordinary filtration if and only if all $w_{i}$ have the same parity.

Proof. If all $w_{i}$ are integers, then $\mathcal{F}_{w}$ is certainly maximal by Lemma 3.5. If they all have odd parity, then we can replace each $w_{i}$ by $w_{i}+1 / 2$ to describe the same filtration but with integer weights. This new weight function is no longer a $*$-weight, but this does not matter.

On the other hand, suppose there exist $w_{i}$ and $w_{j}$ with different parities. Then we have $a<w_{i}-w_{j}<a+1$ for some integer $a$. It follows that $f_{w}(i, j)=a+1$ and $f_{w}(j, i)=-a$. Thus $\mathcal{F}_{w}$ is not a filtration associated to a grading, and consequently $\mathcal{F}_{w}$ is not maximal.

Finally, we obtain the result needed to handle simple Lie algebras of type $\mathrm{B}_{n}, \mathrm{C}_{n}$ and $\mathrm{D}_{n}$. Here, we study $R=\mathrm{M}_{m}(K)$ with $K$ an algebraically closed field and with * an involution of the first kind. By the latter, we mean that the scalar matrices in $R$ are fixed elementwise by the involution.

Lemma 5.7. Let $R=\mathrm{M}_{m}(K)$ be a matrix ring, with $K$ an algebraically closed field of characteristic $\neq 2$, and let $*$ be an involution of $R$ of the first kind. If $\mathcal{F}$ is a $*$-maximal $*$-filtration, then we can write $1=e_{1}+e_{2}+\cdots+e_{m}$ as an orthogonal sum of primitive idempotents with $\mathrm{D}_{m}(K)=\sum_{i=1}^{m} K e_{i} \subseteq F_{0}$. Furthermore, $\left\{e_{1}, e_{2}, \ldots, e_{m}\right\}$ is a $*$-stable set containing at most one $*$-stable idempotent.

Proof. By Theorem 5.5, we can write the matrix ring in such a way that the set $\left\{e_{11}, e_{22}, \ldots, e_{m m}\right\}$ is $*$-stable and that $\mathcal{F}=\mathcal{F}_{w}$ is a $*$-weight filtration. We need only show how to replace pairs of idempotents $e_{i i}$ and $e_{j j}$ that are both $*$-stable by orthogonal primitive idempotents $e$ and $e^{*}$.

To this end, suppose for example that $e_{11}^{*}=e_{11}$ and $e_{22}^{*}=e_{22}$. Then $1^{*}=1$ and $2^{*}=2$, so the condition $w_{i}+w_{i^{*}}=0$ implies that $w_{1}=0=w_{2}$. In particular, $f_{w}(1,2)=0=f_{w}(2,1)$ and hence $F_{0}$ contains the full $2 \times 2$ matrix ring $S=$ $\left(e_{11}+e_{22}\right) R\left(e_{11}+e_{22}\right)$. Furthermore, $S$ is $*$-stable and its diagonal elements are fixed by $*$. With this, we conclude that the action of $*$ on $S$ is given by transpose composed with conjugation by a diagonal matrix. In other words,

$$
\left(\begin{array}{ll}
a & b \\
c & d
\end{array}\right)^{*}=\left(\begin{array}{cc}
a & \lambda c \\
\lambda^{-1} b & d
\end{array}\right)
$$

for some $0 \neq \lambda \in K$. Since $K$ is algebraically closed, there exists $\mu \in K$ satisfying $\mu^{2}=-\lambda$, and we set

$$
f=\left(\begin{array}{cc}
1 / 2 & \mu / 2 \\
\mu^{-1} / 2 & 1 / 2
\end{array}\right)
$$

Then $f$ has rank 1 and trace 1 , so $f$ is an idempotent. Furthermore,

$$
f^{*}=\left(\begin{array}{cc}
1 / 2 & -\mu / 2 \\
-\mu^{-1} / 2 & 1 / 2
\end{array}\right)=1-f
$$

and hence $f f^{*}=0=f^{*} f$.

Thus, there exists a primitive idempotent $e \in\left(e_{11}+e_{22}\right) R\left(e_{11}+e_{22}\right) \subseteq R$ with $e_{11}+e_{22}=e+e^{*}$ and with $e e^{*}=0=e^{*} e$. Since $e$ and $e^{*}$ are contained in $F_{0}$, we can now replace $e_{11}$ and $e_{22}$ by $e$ and $e^{*}$, respectively. Continuing in this manner clearly yields the result. 


\section{Filtrations over ArChimedean ordered GRoups}

Let $R$ be a ring with 1 and let $\mathbb{G}$ be an ordered group, written additively. Then $\mathcal{F}=\left\{F_{i} \mid i \in \mathbb{G}\right\}$ is a $\mathbb{G}$-filtration of $R$ if each $F_{i}$ is an additive subgroup of $R$, $F_{i} F_{j} \subseteq F_{i+j}$ for all $i, j \in \mathbb{G}$, and $F_{i} \subseteq F_{j}$ whenever $i \leq j$. Of course, one also assumes that $\bigcup_{i} F_{i}=R$ and $\bigcap_{i} F_{i}=0$. In particular, $F_{0}$ is a subring of $R$, possibly without 1 , and each $F_{i}$ is an $F_{0}$-bimodule. Again, we say that $\mathcal{F}$ is bounded if there exist $\ell, \ell^{\prime} \in \mathbb{G}$ with $F_{\ell}=0$ and $F_{\ell^{\prime}}=R$.

As in the case when $\mathbb{G}=\mathbb{Z}$, we would like the boundedness of $\mathcal{F}$ to imply that each $F_{i}$ with $i<0$ is nilpotent. This would, of course, follow if for any $i<0$ there exists a positive integer $m$ with $m i \leq \ell$. Thus, if we wish to restrict our study to bounded filtrations that enjoy properties similar to those of $\mathbb{Z}$-filtrations, then it makes sense to assume that $\mathbb{G}$ is an Archimedean ordered group. In this case, one knows from $[\mathrm{Ho}$ that $\mathbb{G}$ is an abelian group, and then from $[\mathrm{Ba}$ that $\mathbb{G}$ is an additive subgroup of the reals $\mathbb{R}$. In particular, either $\mathbb{G} \cong \mathbb{Z}$ or $\mathbb{G}$ is a dense subgroup of $\mathbb{R}$.

Since the $\mathbb{G} \cong \mathbb{Z}$ case has already been considered, we will restrict our attention in this section to dense subgroups of $\mathbb{R}$. Furthermore, since the arguments for dense subgroups are quite similar, and sometimes easier, than those for $\mathbb{Z}$, we will merely stress the differences when they occur, and just sketch the proofs. Note that the obvious analog of Lemma 2.1 guarantees that if $\mathcal{F}=\left\{F_{i} \mid i \in \mathbb{G}\right\}$ is a maximal filtration, then $1 \in F_{0}$.

Lemma 6.1. Let $R$ be a ring and let $\mathbb{G}$ be a dense subgroup of the additive group of real numbers $\mathbb{R}$. If $\mathcal{F}=\left\{F_{i} \mid i \in \mathbb{G}\right\}$ is a maximal bounded filtration of $R$, then $F_{i}=\bigcap_{i^{\prime}>i} F_{i^{\prime}}$. This condition also holds if $R$ is a ring with involution $*$ and $\mathcal{F}$ is a*-maximal $*$-filtration.

Proof. Let $\overline{\mathcal{F}}=\left\{\bar{F}_{i} \mid i \in \mathbb{G}\right\}$ be defined by $\bar{F}_{i}=\bigcap_{i^{\prime}>i} F_{i}$. If $i, j, k \in \mathbb{G}$ with $k>i+j$, then the denseness of $\mathbb{G}$ implies that there exist $i^{\prime}>i$ and $j^{\prime}>j$ with $i+j<i^{\prime}+j^{\prime} \leq k$. With this, it follows that $\bar{F}_{i} \bar{F}_{j} \subseteq \bar{F}_{i+j}$, and $\overline{\mathcal{F}}$ is easily seen to be a bounded filtration of $R$ containing $\mathcal{F}$. Since $\mathcal{F}$ is maximal, the result follows.

We can think of the above as saying that any maximal filtration is upper continuous. Next, we need

Lemma 6.2. Assume that $R$ is a semisimple Artinian ring. If $\mathcal{F}=\left\{F_{i} \mid i \in \mathbb{G}\right\}$ is a maximal bounded filtration of $R$, then $F_{0}$ contains a full diagonal of $R$.

Proof. We consider all right ideal of $R$ of the form $F_{a} R$ with $a \in \mathbb{G}$. Since these form a chain in $R$ and since $R$ has finite composition length, it follows that only finitely many of these ideals can be distinct. Let us denote the distinct members of this chain by $R=I_{0} \supset I_{1} \supset \cdots \supset I_{n} \supset I_{n+1}=0$. We claim that $F_{0} \supseteq I_{i} \cdot 1$. ann $R I_{i+1}$ for all $i=0,1, \ldots, n$. To this end, set $S_{i}=I_{i} \cdot 1 \cdot \operatorname{ann}_{R} I_{i+1}$ and let $r_{i} \in \mathbb{R}$ be defined by $r_{i}=\inf \left\{a \in \mathbb{G} \mid F_{a} R=I_{i}\right\}$.

If $b \in \mathbb{G}$ with $b<0$, then the definition of $r_{i}$ implies that there exists $a \in \mathbb{G}$ with $F_{a} R=I_{i}$ and $a+b<r_{i}$. In particular, $F_{a+b} R$ must equal $I_{k}$ for some $k>i$, and hence $F_{a+b} R \subseteq I_{i+1}$. It follows that $F_{b} I_{i}=F_{b} F_{a} R \subseteq F_{a+b} R \subseteq I_{i+1}$, and thus

$$
\begin{aligned}
S_{i} F_{b} S_{i} & =I_{i} \cdot 1 . \operatorname{ann}_{R} I_{i+1} \cdot\left(F_{b} I_{i}\right) \cdot 1 . \operatorname{ann}_{R} I_{i+1} \\
& \subseteq I_{i} \cdot\left(1 . \operatorname{ann}_{R} I_{i+1} \cdot I_{i+1}\right) \cdot 1 . \operatorname{ann}_{R} I_{i+1}=0 .
\end{aligned}
$$


With this, the proof of Lemma 2.3 shows that $I_{i} \cdot 1 \cdot \operatorname{ann}_{R} I_{i+1}=S_{i} \subseteq F_{0}$, and then, since $I_{0}=R$ and $I_{n+1}=0$, the argument of Lemma 2.5 yields the result.

Now let $R$ be a simple Artinian ring, write $R=\mathrm{M}_{n}(K)$ as the ring of $n \times n$ matrices over a division ring $K$, and let $\mathrm{D}_{n}(K)=\sum_{i=1}^{n} e_{i i} K$ denote the diagonal of $R$. Our next goal is to consider bounded filtrations $\mathcal{F}=\left\{F_{a} \mid a \in \mathbb{G}\right\}$ with $F_{0} \supseteq \mathrm{D}_{n}(K)$. As usual, we call these diagonal filtrations, and we define the real valued degree function $f$ of $\mathcal{F}$ by

$$
f(i, j)=\inf \left\{a \in \mathbb{G} \mid e_{i j} \in \mathcal{F}_{a}\right\}
$$

for all $i, j=1,2, \ldots, n$.

Lemma 6.3. Let $\mathcal{F}=\left\{F_{a} \mid a \in \mathbb{G}\right\}$ be a diagonal filtration of $R=\mathrm{M}_{n}(K)$ and let $f$ be its degree function. Assume that $\mathcal{F}$ is upper continuous.

(i) For each $a \in \mathbb{G}$, we have $F_{a}=\sum e_{i j} K$ where the sum is over all $i, j$ with $f(i, j) \leq a$. In particular, $f$ uniquely determines the filtration.

(ii) $f(i, j)+f(j, k) \geq f(i, k)$ for all $i, j, k=1,2, \ldots, n$.

(iii) If $\mathcal{G}=\left\{G_{a} \mid a \in \mathbb{G}\right\}$ is an upper continuous diagonal filtration with degree function $g$, then $\mathcal{G}$ contains $\mathcal{F}$ if and only if $g(i, j) \leq f(i, j)$ for all $i, j$.

Proof. We begin with part (i). Since $F_{0} \supseteq D_{n}(K)$ and since $F_{a}$ is an $F_{0}$-bimodule, we know that $F_{a}$ is a suitable sum of $e_{i j} K$ terms. Furthermore, by definition of the degree function $f$, it is clear that $e_{i j} K$ does not occur in $F_{a}$ if $a<f(i, j)$. On the other hand, suppose $a \geq f(i, j)$ and let $a^{\prime} \in \mathbb{G}$ with $a^{\prime}>a \geq f(i, j)$. Then, again by the definition of $f$, we have $e_{i j} K \subseteq F_{a^{\prime}}$, and upper continuity yields the result. Parts (ii) and (iii) follow as in Lemma 3.1 .

Next, we introduce the weight filtrations. Here the weight functions $w$ are real valued, rather than integer valued, and we define $\mathcal{F}_{w}=\left\{F_{a} \mid a \in \mathbb{G}\right\}$ by $F_{a}=$ $\sum e_{i j} K$, where the sum is over all $i, j$ with $w_{i}-w_{j} \leq a$. If $f_{w}$ is the degree function of $\mathcal{F}_{w}$, then the denseness of $\mathbb{G}$ easily implies that $f_{w}(i, j)=w_{i}-w_{j}$ for all $i, j$. Of course, two weight functions $v$ and $w$ are said to be equivalent if $v_{i}-w_{i}=v_{j}-w_{j}$ for all $i, j$, and equivalent weights give rise to the same filtration. In view of the above work and the proof of Lemma 3.4, we have

Lemma 6.4. Let $\mathcal{F}=\left\{F_{a} \mid a \in \mathbb{Z}\right\}$ be an upper continuous diagonal filtration of $\mathrm{M}_{n}(K)$ with degree function $f$. Then there exists a real valued weight function $w$ with $f(i, j) \geq w_{i}-w_{j}=f_{w}(i, j)$ for all $i, j$. In particular, $\mathcal{F}$ is contained in the weight filtration $\mathcal{F}_{w}$.

The methods of Lemma 3.5 and Theorem 3.6 now combine to yield

Theorem 6.5. Let $R$ be a semisimple Artinian ring, let $\mathbb{G}$ be a dense subgroup of $\mathbb{R}$, and let $\mathcal{F}=\left\{F_{i} \mid i \in \mathbb{G}\right\}$ be a bounded $\mathbb{G}$-filtration of $R$.

(i) If we write $R=R_{1} \oplus R_{2} \oplus \cdots \oplus R_{k}$ as a finite direct sum of simple Artinian rings, then $\mathcal{F}$ is maximal if and only if it is a sum $\mathcal{F}=\mathcal{F}_{1} \oplus \mathcal{F}_{2} \oplus \cdots \oplus \mathcal{F}_{k}$, where each $\mathcal{F}_{j}$ is a maximal filtration of $R_{j}$.

(ii) If $R$ is simple, then $\mathcal{F}$ is maximal if and only if there exists a suitable description of $R$ as a full matrix ring over a division ring such that $\mathcal{F}$ becomes a weight filtration of $R$. Furthermore, two weight filtrations are equal if and only if their corresponding weights are equivalent. 
In terms of rings with involution, there is even less to do. Assume that $R=$ $\mathrm{M}_{n}(K)$ is an $n \times n$ matrix ring over the division ring $K$ and let $*$ be an involution defined on $R$. As before, fix matrix units $e_{i j}$ for $i, j=1,2, \ldots, n$ and assume that the set $\left\{e_{11}, e_{22}, \ldots, e_{n n}\right\}$ is $*$-stable. Again, we define a corresponding map $*$ on the subscripts $\{1,2, \ldots, n\}$ by setting $e_{i i}^{*}=e_{i^{*} i^{*}}$, and we say that the real valued weight function $w$ is a $*$-weight if $w_{i}+w_{i^{*}}=0$ for all $i=1,2, \ldots, n$. It follows easily that the terms of the weight filtration $\mathcal{F}_{w}$ determined by the $*$-weight $w$ are all $*$-stable, so $\mathcal{F}_{w}$ is a $*$-filtration. The main result in this context is

Theorem 6.6. Let $R$ be a simple Artinian ring of characteristic $\neq 2$ admitting an involution $*$, let $\mathbb{G}$ be a dense subgroup of $\mathbb{R}$, and let $\mathcal{F}=\left\{F_{i} \mid i \in \mathbb{G}\right\}$ be a bounded $*$-filtration of $R$. Then $\mathcal{F}$ is $*$-maximal if and only if there exists a suitable description of $R$ as a full $n \times n$ matrix ring over a division ring with $\left\{e_{11}, e_{22}, \ldots, e_{n n}\right\}$ stable under the action of $*$ and with $\mathcal{F}=\mathcal{F}_{w}$ being a $*$-weight filtration of $R$. Furthermore, two *-weight filtrations are equal if and only if their corresponding weights are identical.

Observe that, in the situation where $\mathbb{G}$ is a dense subgroup of $\mathbb{R}$, all $*$-weights are ordinary weights and hence all *-weight filtrations are maximal as ordinary filtrations. Finally, the argument of Lemma 5.7 applies to yield

Lemma 6.7. Let $R=\mathrm{M}_{m}(K)$ be a full matrix ring with $K$ an algebraically closed field of characteristic $\neq 2$, and let $*$ denote an involution of $R$ of the first kind. If $\mathcal{F}$ is a $*$-maximal $*$-filtration over a dense subgroup $\mathbb{G}$ of $\mathbb{R}$, then we can write $1=e_{1}+e_{2}+\cdots+e_{m}$ as an orthogonal sum of primitive idempotents with $\mathrm{D}_{m}(K)=$ $\sum_{i=1}^{m} K e_{i} \subseteq F_{0}$. Furthermore, $\left\{e_{1}, e_{2}, \ldots, e_{m}\right\}$ is a $*$-stable set containing at most one $*$-stable idempotent.

\section{REFERENCES}

[Ba] R. Baer, Zur Topologie der Gruppen, J. Reine Angew. Math. 160 (1929), 208-226.

[B] Y. Barnea, Maximal graded subalgebras of loop toroidal Lie algebras, Algebras Represent. Theory, to appear.

[BP] Y. Barnea and D. S. Passman, Filtrations in semisimple Lie algebras, Trans. Amer. Math. Soc., submitted.

[H] I. N. Herstein, Rings with Involution, Univ. Chicago Press, Chicago, 1976. MR0442017 $(56: 406)$

[Ho] O. Hölder, Die Axiome der Quantität und die Lehre vom Mass, Ber. Verh. Sächs. Ges. Wiss. Leipzig. Math.-Phys. Kl. 53 (1901), 1-64.

[ZS] M. V. Zaicev and S. K. Sehgal, Finite gradings of simple Artinian rings, Moscow Univ. Math. Bull 3 (2001), 21-24. MR.1863551 (2002e:16067)

Department of Mathematics, University of Wisconsin, Madison, Wisconsin 53706

E-mail address: passman@math.wisc.edu 